\title{
Social Networks Community Detection Using the Shapley Value
}

\author{
Alireza Hajibagheri, Hamidreza Alvari, Ali Hamzeh, Sattar Hashemi \\ Computer Science and Engineering \\ Shiraz University \\ Shiraz, Iran \\ \{hajibagheri, alvari, ali\}@cse.shirazu.ac.ir, s_hashemi@shirazu.ac.ir
}

\begin{abstract}
By increasing the popularity of social networking websites like Facebook and Twitter, analysis of the structure of these networks receives significant attentions. The most important part of these analyses is towards detecting communities. The aforementioned structures are known with extremely high inter-connections versus few intra-connections in the graphs. In this paper, we have addressed the community detection problem by a novel framework based on Information Diffusion Model and Shapley Value Concept. Here, each node of the underlying graph is attributed to a rational agent trying to maximize its Shapley Value in the form of information it receives. Nash equilibrium of the game corresponds to the community structure of the graph. Compared with other methods, our approach demonstrates promising results on the well-known real world and synthetic graphs.
\end{abstract}

Keywords-social network; shapley value; information diffusion; Nash equilibrium; community structure

\section{INTRODUCTION}

Detecting communities in graphs, is attracting increasing interest, with numerous applications such as those corresponding to finding related topics within World Wide Web [1], friend suggestion in social networks like Facebook [2] and discovering functional groupings in metabolic networks [3]. Furthermore, finding clusters of similar customers in the purchase network (e.g. www.amazon.com) enhances recommendation systems performance [4].

A social network is a complex graph-based structure composed of individuals called nodes, which are connected by one or more specific types of interdependency such as friendship, common interest, financial exchange, etc. which are often called edges. Recently, online social websites like Facebook and Twitter have been receiving most attentions and become very popular among cyberspace users, since they provide users an environment to chat, send email, spread opinions and etc., easily without a need to physical meetings. As a result, the way that Social Network Analysis (SNA) deals with, has been changed widely.

Like many complex networks, social networks demonstrate lots of interesting properties among which we can mention network transitivity [5], power law degree distributions [6] and the well-known property community structure [7]. In its simplest form, community structure refers to the existence of modules or communities with dense interconnections versus sparse intra-connections. A toy example network and its community structure are shown in Fig. 1.
During last decade, a large variety of algorithms have been proposed to solve the problem of community detection. However, most of them work based on the structural attributes of the network such as number of vertices, degree of each vertex, etc. [8]. In this work we address the community detection problem as a game-theoretic approach employing Information Diffusion model and Shapley Value. As results show, our proposed framework performs well in detecting finer community structure of the underlying graph.

The rest of the paper is organized as follows: in Section II, a brief review of the state-of-the-art methods is presented. Then, in Section III, we describe our proposed framework in detail. Our results on different real-world and synthetic datasets are described in Section IV. We conclude the paper in Section V.

\section{RELATED WORK}

The problem of community detection is a long standing research appeared in various forms in several disciplines including sociology and computer science. The first analysis of community structure dates back to 1955 and the work carried out by Weiss and Jacobson [9] in which they searched for workgroups within a government agency. However, research on communities actually started even earlier than this work. In 1927, Stuart Rice tried to look for clusters of people in small political bodies, based on the similarity of their voting patterns [10]. Traditional techniques to find communities in social networks are hierarchical and partitional clustering, where vertices are joined into groups according to their mutual similarity.

Several works have been done in the literature which can be categorized into two main groups: optimization methods and methods with no optimization, which search for some predetermined structures. From these methods one can refer to the works done by Girvan and Newman in 2002 and 2004 introducing two important concepts 'edge betweenness' [7] and 'modularity' [11], the work of Brandes and Erlebach which coins the term 'conductance' [12] and the work done by Palla et al. [13]. In [7], Girvan and Newman proposed a new algorithm to identify edges lying between communities which by their successive removal, the isolation of the communities happens. The inter-community edges are detected according to the values of a centrality measure, the edge betweenness that expresses the importance of the role of the edges in processes where signals are transmitted across the graph following paths of minimal length. That work 
triggered a big activity in the field where many new methods have been proposed in recent years [8]. In particular, physicists entered the game, bringing in their tools and techniques such as spin models, optimization, percolation, random walks, synchronization and etc., which rapidly became the main ingredients of new original algorithms. The field has also taken advantages of concepts and methods from computer science, nonlinear dynamics, sociology and discrete mathematics.

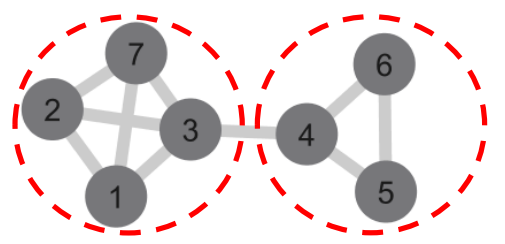

Fig. 1. Toy example network and its community structure (Each circle with dotted border shows a community)

\section{PROPOSED METHOD}

\section{A. Motivation}

It is necessary for everyone to make friendship with those who have something valuable to share with. Informative human-human social interactions motivated us to assign the community formation problem in social networks to a play of interactions between their constituents, i.e. people.

Game theory is a good tool to capture both the behavior of individuals and strategic interactions among them [14], because it can model strategic interactions between rational, autonomous and intelligent agents mathematically. In this study, based on the work done by Alvari et al. [15] we consider the community detection problem as a game in a multiagent environment and ascribe each vertex of the underlying graph to an agent. These agents try to form communities based on their Shapley values by joining to communities with total useful information for them. To quantify the information exchanged between agents, we use the formulation in [16]. Finally, the Nash equilibrium of the game corresponds to the clustering of the network. In the next, we first explain the information diffusion concept used in our framework and then our game-theoretic approach is explained in detail.

\section{B. Information Diffusion in Social Networks}

Diffusion processes take place in social networks and we can use this processes to model different phenomena in the world surrounding us such as the spread of computer and human viruses, and the information about an invention or idea. Obtaining accurate information about these processes is difficult; therefore, we will use diffusion models to describe their behavior. A diffusion model probabilistically indicates a process of diffusion takes place and spreads through a social network [16].

Information diffusion is a special kind of diffusion process in which information spreads through vertices of a social network. Here, information is considered as specific details about an innovation or an idea. There exist two wellknown probabilistic models for information diffusion, the Independent Cascade model and the Linear Threshold model
[17][18][19]. Recently, Lahiri and Cebrian [16] proposed a new model based on canonical Genetic Algorithm [20] paired with Holland's hyper-defined objective functions [21], namely GADM (Genetic Algorithm Diffusion Model). In the followings formal definition of the diffusion model is given.

Definition 1. A diffusion model maps current state vector of a vertex to a new state vector. Given a graph $G=(V, E)$ and a state vector $S_{v}^{(t)}$ for every vector $v \in V$ at time $t$, this model outputs a new state vector $S_{v}^{(t+1)}$ for every vertex at time $t+1$ based on the state of all interacting vertices [16].

The GA Diffusion Model (GADM) [16]. We now explain the GADM which we will use in our proposed community detection algorithm. Lahiri and Cebrian showed that canonical Genetic Algorithm, which uses binary string chromosomes and one-point crossover, can be used as a model for information diffusion process. Their work deals with dynamic social networks where a set of vertices $V=\left\{v_{1}, \ldots, v_{n}\right\}$ interacts over $T$ time periods. Furthermore, a mapping exists between these individuals and chromosomes in the GA population. Chromosomes are state vectors of individuals at each time step $t$. These state vectors are binary string with length $\beta$. Initial state vectors can be set to zero or one can use a random distribution to initialize them. Additionally, an objective function $f(x)$ which assigns a score to each vertex state vector is needed. For each edge $(u, v)$ in the social network at time step $t$, logic of canonical genetic algorithm is applied to the corresponding chromosomes in the GA population. GADM algorithm is shown in Algorithm 1. In this algorithm, state vectors are modified and adopted based on crossover operator and objective function, resulting to the occurrence of diffusion process in the social network. Obviously, we have a missing component here, and that would be an objective function to bring meaning to the mapping between the state vectors of a node and chromosomes in GA population.

\footnotetext{
Algorithm 1. GADM

1. Input: Initialize state vectors of nodes $u$ and $v$ to $S_{v}$ and $S_{u}$.

2. Output: New state vector for nodes $u$ and $v$.

3. Repeat \{

4. $\quad$ Set $S_{v}^{(t+1)}=S_{v}^{(t)}$ and $S_{u}^{(t+1)}=S_{u}^{(t)}$.

5. Select a random crossover point $\mathrm{c}$ between $[1, \beta]$.

6. Create $y_{1}$ and $y_{2}$ by swapping the tails of $S_{v}^{(t)}$ and $S_{u}^{(t)}$ where the tail is defined as all positions including and after index c.

7. Update state vectors: $S_{v}^{(t+1)}=\operatorname{argmax}_{x=\left\{s_{v}^{t}, y_{1}, y_{2}\right\}} f(x)$ and $S_{u}^{(t+1)}=\operatorname{argmax}_{x=\left\{s_{u}^{t}, y_{1}, y_{2}\right\}} f(x)$

8. $\quad\}$

9. Until (All interactions are checked)
}

To fill the aforementioned gap, Holland hyper-defined objective functions (HDFs) [21] which are synthetic objective functions can be used in GADM algorithm. HDFs 
are constructed from a predefined schema, a short substring with wildcards starting at a specific position. Schemas take place at random positions within the strings. At first, schemas are relatively short and we call them order 1 schemas. Pairs of such schema are concatenated to generate order 2 schema and so on. Moreover, each schema receives an individual positive or negative score generated randomly from some range. At this point, HDFs take binary strings as input and return an objective score that is the sum of the scores of all the individual schemas it contains [16].

The following is an example demonstrating the generation of a simple HDF which takes a binary string of length $n=10$ as an input. The asterisk ('*') shows a character that matches both zero and one ('don't care' character).

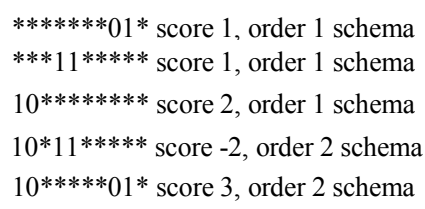

In GADM, each schema is a 'unit' of information and different schema carries different values. As we mentioned before, every individual in a social network has a state vector. These state vectors can contain different schema's, which means every individual carries certain pieces of information. Each time a pair of nodes interacts with each other, they randomly exchange information based on the crossover operator. This process could result in either both nodes gaining more information than they had before or no benefit from interaction.

There is a key difference between GADM and earlier studies on information diffusion such as [17] [18] [19]. GADM is able to model the propagation and spread of multiple 'units' of information from individual to individual. These different units can interact in non-linear and complex ways based on a randomly generated HDF in order to affect the total 'information value' of each individual. The objective is to estimate the average information value of each node after multiple random HDFs and state vector initializations, in order to determine whether every individual in our social network is positioned to receive the same amount of information as a result of their interactions with other individual.

Lahiri and Cebrian [16] used GADM to model information flow between people as they exchange emails. To test their method they used Enron email dataset. Their results indicated that only a small portion of vertices in a social network receive more information than others, regardless of how much they start with. This term is called 'information elitism' and they showed that it is not simply related to a trivial network property such as degree. To put it simply, one might assume that a vertex with high income degree may contain more information than others, but the correlation between the final information value of nodes and their degree shows absolutely the opposite. As a result, a community detection method based on this feature, is independent from network structure.

In order to use this feature in our method, we have to save information that is exchanged between nodes. Each node can either receive information from others or give them new units of information through crossover operation. Since we apply our method on static social networks, the interpretations of $S_{v}^{(t)}$ and $S_{v}^{(t+1)}$ are different from what is actually used in GADM. Here, $S_{v}^{(t)}$ stands for the state vector of node $v$ before an interaction with node $u$ while $S_{v}^{(t+1)}$ is the state vector of node $v$ after the interaction with node $u$.

To avoid the bias of randomness we run multiple trials with different HDFs and state vectors initialization. In each run, we store the information exchanged between two nodes in a matrix called Information Matrix I. These values are normalized with respect to the maximum exchanged value after each run. What we need over multiple trials is the average of these values which we call 'average normalized information' or ANI:

$$
\boldsymbol{I}=\frac{\boldsymbol{I}^{(1)}+\cdots+\boldsymbol{I}^{(n)}}{n}=\left[\begin{array}{ccc}
\frac{I_{11}^{(1)}+\cdots+I_{11}^{(n)}}{n} & \cdots & \frac{I_{1 n}^{(1)}+\cdots+I_{1 n}^{(n)}}{n} \\
\vdots & \ddots & \vdots \\
\frac{I_{n 1}^{(1)}+\cdots+I_{n 1}^{(n)}}{n} & \cdots & \frac{I_{n n}^{(1)}+\cdots+I_{n n}^{(n)}}{n}
\end{array}\right]
$$

where superscripts show the information matrix of $i^{\text {th }}$ iteration and $I$ is the final Information Matrix.

TABLE I. DEFINITIONS OF SYMBOLS

\begin{tabular}{|ll|}
\hline Sym. & Definition \\
\hline$G$ & Undirected and unweighted graph \\
\hline$n, m$ & Number of nodes and edges \\
\hline$S$ & Set of strategies of all agents \\
\hline$s_{i}$ & Strategy of agent $i$ \\
\hline$\Phi_{i}$ & Shapley value of agent $i$ \\
\hline $\mathbf{I}$ & Information Matrix \\
\hline$I_{i j}$ & Information that agent $i$ received from agent $j$ \\
\hline$\delta_{i j}$ & Equals 1 if agents $i$ and $j$ are in the same community \\
\hline
\end{tabular}

\begin{tabular}{ll}
\hline Algorithm 2.SID \\
\hline 1. & Input: underlying network graph $G$. \\
2. & Output: community as a final division of $G$. \\
3. Initialize each node of $G$ as an agent. \\
4. Initialize community as a set of all communities. \\
5. I = GADM $(G) / /$ Create Information Matrix \\
6. Repeat \{ \\
7. Choose a random agent from pool of agents. \\
8. Choose the best operation among join, leave, switch or no operation \\
9. $\quad$ according to (4). \\
10. Until (local Nash equilibria is reached) \\
\hline
\end{tabular}




\section{Framework}

In this section, we first introduce notations and parameters which are used in our work in Table I.

Regarding what has been discussed so far, we put each vertex down to a rational agent who just thinks about maximizing its Shapley value. In doing so, whenever it is selected from a pool of agents, periodically makes personal decisions while it is involved in the game. Later on this agent decides whether to join a new community, leave one of its communities or switch from a community to a new one.

After all, new Shapley value for the selected agent is calculated according to (2) and its old value is replaced by the new one.

$$
v_{i}(S)=\frac{1}{m} \sum_{j=1, j \neq i}^{n} I_{i j} \delta_{i j}
$$

where $v: 2^{C} \rightarrow \mathbb{R}$ is a characteristic function that assigns a value to each subset of $C$. Here $C$ is a community which consists of one or more nodes. For this agent the Shapley value is defined as:

$$
\Phi_{i}(v)=\frac{1}{n !} \sum_{\pi \epsilon \Omega}\left[v\left(P_{i}(\pi) \cup i\right)-v\left(P_{i}(\pi)\right)\right]
$$

where $\Omega$ is the power set over $C$ and $P_{i}(\pi)$ is the set of players appearing before the $i$ th player in set $\pi$. In this framework, the best response strategy of an agent $i$ with respect to the strategies $S_{-i}$ of other agents is calculated by:

$$
\operatorname{argmax}_{s_{i}^{\prime} \subseteq[k]} \Phi_{i}\left(S_{-i}, s_{i}^{\prime}\right)
$$

The strategy profile $S$ forms a pure Nash equilibrium of the community formation game if all agents play their best strategies. In other words, in Nash equilibrium no agent can improve its own utility by changing its strategy; that is each agent is satisfied with the current utility:

$$
\forall i, s_{i}^{\prime} \neq s_{i}, \Phi_{i}\left(S_{-i}, s_{i}^{\prime}\right) \leq \Phi_{i}\left(S_{-i}, s_{i}\right)
$$

Since reaching global Nash equilibrium is not feasible in this game, we used local Nash equilibrium [22]. The strategy profile $S$ forms a local equilibrium if all agents play their local optimal strategies. Here $l s\left(s_{i}\right)$ refers to local strategy space of agent $i$ :

$$
\forall i, s_{i}^{\prime} \in l s\left(s_{i}\right), \Phi_{i}\left(S_{-i}, s_{i}^{\prime}\right) \leq \Phi_{i}\left(S_{-i}, s_{i}\right)
$$

Finally the algorithm of the proposed method, namely SID, is shown in Algorithm 2.

\section{EXPERIMENTS}

We have mentioned earlier that the proposed approach can perform well in detecting communities in social networks. To illustrate this, we now present our experimental results on the well-studied real-world datasets and two popular synthetic networks. We compare our algorithm with five decentralized algorithms and two centralized algorithms. We use two evaluation metrics to show the performance of our algorithm: Normalized Mutual Information (NMI) [23] and Modularity $Q$ [24]. Table II shows algorithms and parameter settings we have used for our experiments respectively. We have implemented all of the algorithms in JAVA on a system with $4 \mathrm{G}$ of RAM and Intel CPU 2.53 $\mathrm{GHz}$ for the purpose of fair comparison.

TABLE II. ALGORITHMS USED FOR EXPERIMENTS AND THEIR PARAMETERS $(\mathrm{D}=$ DECENTRALIZED, $\mathrm{C}=\mathrm{CENTRALIZED})$

\begin{tabular}{|c|c|c|}
\hline Algorithm & Type & Parameters \\
\hline HA [31] & $\mathrm{D}$ & $m=0.1, \delta=0.05$ \\
\hline MMC [32] & $\mathrm{D}$ & $\alpha=2.65, \beta=2, \rho=0.9, \mu=1.08, \eta=0.7, \gamma=0.1$ \\
\hline InfoMap [10] & $\mathrm{C}$ & None \\
\hline LPA [33] & $\mathrm{D}$ & None \\
\hline
\end{tabular}

Sharc and HA are different varieties of basic label propagation methods while MMC is a label propagation method which uses labels to show information. InfoMap is a centralized method which is based on "unfolding" technique.

\section{A. Evaluation}

1) Normalized Mutual Information (NMI) [23]: We often use normalized mutual information to measure the similarity between found partition and the ground trustpartition. Assume that we have two partitions called $\mathrm{P}$ and Q. We form a matrix $\mathbf{N}$ in $|P| \times|Q|$.

Element $N_{i j}$ of the matrix $\mathbf{N}$ shows the number of common nodes of the $\mathrm{i}^{\text {th }}$ community of $P$ and $\mathrm{j}^{\text {th }}$ community of $Q$. In addition, we show the sum of $i^{\text {th }}$ row with $N_{i}$ and the sum of the $\mathrm{j}^{\text {th }}$ column with $N_{. j}$. NMI is in range $[0,1]$ and it is defined as follow:

$$
I(P, Q)=\frac{-2 \sum_{i=1}^{|P|} \sum_{j=1}^{|Q|} N_{i l} \log \left(\frac{N_{i j} N}{N_{i .} N_{. j}}\right)}{\sum_{i=1}^{|P|} N_{i .} \log \left(\frac{N_{i .}}{N}\right)+\sum_{j=1}^{|Q|} N_{. j} \log \left(\frac{N_{. j}}{N}\right)}
$$

2) Modularity [24]: As we stated above, we can use NMI only when the ground trust structure is provided. However, in some special cases, we have to test the algorithm on some synthetic datasets with no gound trust and that's when modularity will come in handy.

Although this measure has drawbacks and becomes unreliable when our networks are too sparse [25], modularity is the most popular qualitative measure in detecting communities in social networks. Formaly modularity is defined as follows:

$$
Q=\sum_{s=1}^{k}\left[\frac{l_{s}}{L}-\left(\frac{d_{s}}{2 L}\right)^{2}\right]
$$

where $k$ is the number of revealed communities, $L$ denotes the total number of edges, and $d_{s}$ is the sum of degrees of nodes in community $S$. 


\section{B. Dataset}

We explain our results on two well-known real-world benchmark networks and three synthetic networks in this section. Experimental results on these datasets demonstrate that our approach performs well, both on real-world and synthetic datasets.

\section{1) Benchmark Networks:}

a) Dolphin Network: The dolphin network represents the relationships of 62 bottlenose dolphins, introduced by Lusseau [26] consisting of 62 nodes and 159 edges. Based on Lusseau observations, these dolphines were divided into two groups because of some reasons. The performances of a variety of algorithms on this network in the form Modularity and NMI is depicted in Table III.

TABLE III. The Average Results OF 100 Runs By SID, HA, MMC, LPA AND INFOMAP

\begin{tabular}{|c|c|c|c|c|}
\hline & \multicolumn{2}{|c|}{ Q } & \multicolumn{2}{c|}{ NMI } \\
\hline & Dolphin & Karate & Dolphin & Karate \\
\hline SID & $\mathbf{0 . 5 3 8}$ & $\mathbf{0 . 3 7 3}$ & $\mathbf{0 . 7 1 5}$ & $\mathbf{1 . 0 0}$ \\
\hline HA & 0.449 & 0.300 & 0.707 & 0.754 \\
\hline MMC & 0.526 & 0.371 & 0.579 & 1.00 \\
\hline LPA & 0.450 & 0.362 & 0.710 & 0.751 \\
\hline InfoMap & 0.514 & 0.354 & 0.695 & 0.643 \\
\hline
\end{tabular}

As it can be understood, SID performs better than other methods on this dataset. Results shown in Table III are average from 100 runs.

b) Zachary Karate Club Network: The Zachary karate club network [27] which consists of 34 nodes and 78 edges shows relations between these nodes standing for club members. This network is widely used as a benchmark for community detection algorithms. The Zachary karate club network is divided into two roughly equal-sized groups due to the disagreement between club administrator and the principal karate teaher, represented by node 34 and node 1 respectively. Our approach dicloses these two groups perfectly.

\section{2) Synthetic Networks:}

a) GN Synthetic Networks: GN synthetic network is probably the most popular community generation model [28]. This model contains four communities and 128 nodes in which each node has a same expected degree-16. There exists a mixing parameter $\mu$ which controls the ratio between the external degree with respect to community and degree of a node. The results shown in Fig. 2 are the performances of SID, MMC, LPA, HA and InfoMap. Every point in this figure is the average of 100 runs for each algorithm.

The number of communities is fixed to four in GN sythetic networks and our algorithm detects only four communities in each run while other algorithms such as Sharc finds more than four communities sometimes. As we can see in Fig.2, the proposed approach performs outstanding when the value of mixing parameter is lower than 0.45 . Finally the NMI value approaches to zero when we set the mixing parameter to 0.5 similar to HA and LPA.

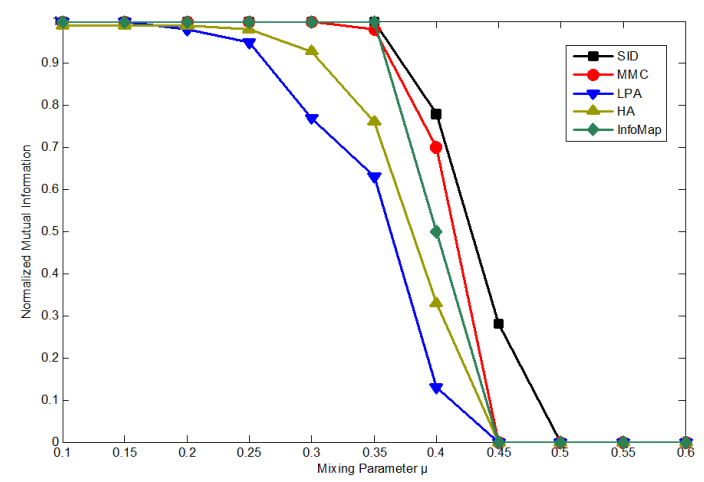

Fig. 2. Algorithms Performances on GN Synthetic Network

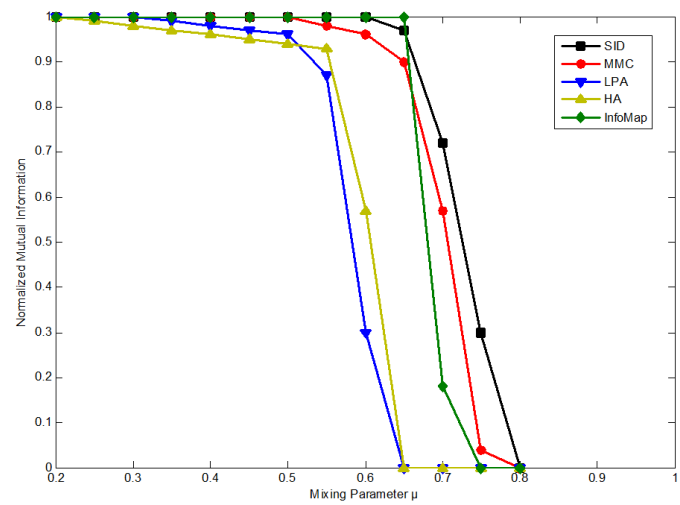

(a) Small communities (20-50) - 1000 nodes

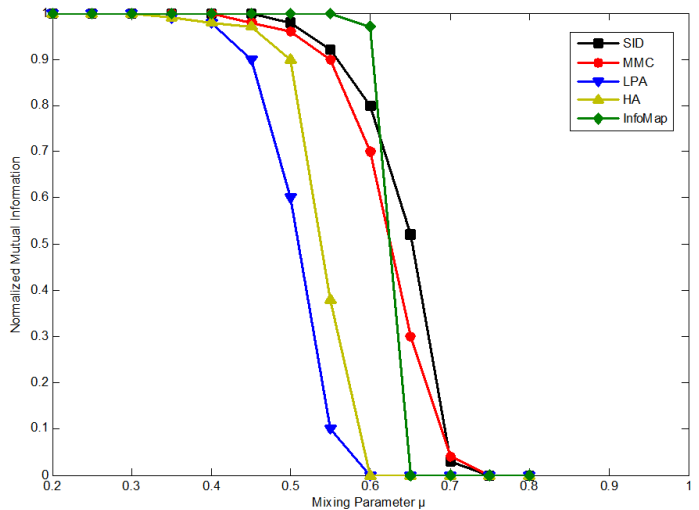

(b) Big communities (20-100) - 1000 nodes

Fig. 3. Algorithms Performances on LFR Synthetic Network.

b) LFR Synthetic Networks: Recently Lancichinetti [29] proposed a method to generate synthetic networks in which we can control both, the distribution of degrees and communities sizes. This attribute of LFR method covers the GN synthetic networks drawbacks and as a result it is becoming a commonly used method to generate synthetic networks. It is realistic, reasonable and also able to generate networks with overlapping communities. For this experiment, the maximum degree of each node is 50 and the 
average degree is 20 . The exponent of the degree distributon is set to -2 . Communities have between 20 to 50 nodes and 20 to 100 nodes and we call them small communities and big communities respectively. The performances of different algorithms compared with our algorithm, applied on two different networks with small and big communities is shown in Fig. 3. As it can be seen in this figure, SID performs better than almost all the other methods when $\mu \leq 0.7$ while LPA and HA reach zero at $\mu=0.65$. When $\mu>0.75$, nearly all of algorithms perform equally.

\section{CONCLUSION AND FUTURE WORK}

In this study, we have proposed a Game theoretic framework based on Shapley value of the vertices and Information Diffusion Model to identify community structure of the underlying network graph. In this framework each node of the graph is considered as an agent who calculates Shapley value for each community it has connection with. Specifically, each selected agent chooses between join, leave and switch operations iteratively. The Nash equilibrium of the game corresponds to the community structure of the network.

The results demonstrate our method's superiority over other well-known methods. For future work, the proposed framework can be easily extended to be used in dynamic social networks. In addition, different models for information diffusion in social networks can be used.

\section{REFERENCES}

[1] Flake G W, Lawrence S R, Giles C L, Coetzee F M. IEEE Computer 35, 2002, 66-71.

[2] M. Girvan and M.E.J. Newman, "Community structure in social and biological networks," in Proceedings of the National Academy of Sciences,vol. 99, no. 12, 2002, pp. 7821-7826.

[3] Chen J, Yuan B. Bioinformatics 22(18),2006,2283.

[4] Reddy K P, Kitsuregawa M, Sreekanth P, Rao S S. In DNIS '02: Proceedings of the Second International Workshop on Databases in Networked Information Systems (Springer-Verlag, London, UK), 2002, pp. 188-200.

[5] Watts D J, Strogatz S H. Nature 393,1998, 440-442.

[6] Barabási A-L, Albert R. Science 286, 1999, 509-512.

[7] Newman M E J. SIAM Rev., 2003,45(2),167.

[8] Fortunato S. Community detection in graphs. arXiv:0906.0612, 2009.

[9] Weiss R S, Jacobson E. Am. Sociol. Rev.20, 1955, 661.

[10] M. Rosvall and C.T. Bergstrom, "Maps of random walks on complex networks reveal community structure," Proceedings of the National Academy of Sciences, vol. 105, no. 4, pp. 1118-1123, 2008.

[11] Newman M E J, Girvan M. Phys. Rev. E69 (2), 2004, 026113.

[12] Brandes U, Erlebach T. Network analysis :methodological foundations. Springer Verlag, Berlin, 2005.

[13] Palla G, Derényi I, Farkas I, Vicsek T. Uncovering the Overlapping Community Structure of Complex Networks in Natureand Society. Nature 435, 2005, 814-818.

[14] Adjeroh D, Kandaswamy U. Game-Theoretic Analysis of Network Community Structure. 2007, Vol.3, No.4, pp. 313-325, doi:10.5019/j.ijcir.2007.112.

[15] Alvari, H., Hashemi, S., Hamzeh, A.: 'Detecting Overlapping Communities in Social Networks by Game Theory and Equivalence Concept'. AICI 2011, Part II, LNAI 7003, pp.620 - 630, 2011, Springer-Verlag
[16] M. Lahiri and M. Cebrian. The genetic algorithm as a general diffusion model for social networks. Proc. of the 24th AAAI Conference on Artificial Intelligence (AAAI 2010), Atlanta, Georgia, 2010.

[17] D. Gruhl, R. Guha, D. Liben-Nowell, and A. Tomkins, "Information diffusion through blogspace," SIGKDD Explorations, vol. 6, pp. 491501,2004 .

[18] D. Kempe, J.M. Kleinberg, and É. Tardos, "Maximizing the spread of influence through a social network," in Proceedings of the ninth ACM SIGKDD international conference on Knowledge discovery and datamining, ser. KDD ’03, 2003, pp. 137-146

[19] T. Fushimi, T. Kawazoe, K. Saito, M. Kimura, and H. Motoda, "What does an information diffusion model tell about social network structure?"in Pacific Rim Knowledge Acquisition Workshop, 2008, pp. 122-136.

[20] Goldberg, D. E. 1989. Genetic algorithms in search, optimization and machine learning. Addison-Wesley Longman Publishing Co., Inc. Boston, MA, USA.

[21] Holland, J. H. 2000. Building blocks, cohort genetic algorithms, and hyperplane-defined functions. Evol. comp.8(4):373-391.

[22] Alós-Ferrer C, Ania A. Local equilibria in economic games. 2001, Econ Lett 70 (2), 165-173.

[23] L. Danon, A. Diaz-Guilera, J. Duch, and A. Arenas, "Comparing community structure identification," Journal of Statistical Mechanics:Theory and Experiment, vol. 9, p. 8, 2005.

[24] M.E.J. Newman and M. Girvan, "Finding and evaluating community structure in networks," Phys. Rev. E, vol. 69, no. 2, p. 026113, 2004.

[25] S. Fortunato and M. Barthélemy, "Resolution limit in community detection," in Proceedings of the National Academy of Sciences, vol.104, no. 1. National Acad Sciences, 2007, p. 36

[26] D. Lusseau, "The emergent properties of a dolphin social network," in Proceedings of the National Academy of Sciences, vol. 270 Suppl, no.0962-8452, 2003, pp. S186-8.

[27] W. Zachary, "An information flow model for conflict and fission in small groups," Journal of Anthropological Research, vol. 33, pp. 452473,1977

[28] A. Lancichinetti and S. Fortunato, "Community detection algorithms: a comparative analysis," Phys. Rev. E, vol. 80, no. 5, p. 056117, 2009.

[29] Lancichinetti A, Fortunato S. Benchmarks for testing community detection algorithms on directed and weighted graphs with overlapping communities. Phys RevE 80(1): 16118, 2009.

[30] G.J. Herbiet and P. ,Bouvry, "Sharc: Community-based partitioning for mobile ad hoc networks using neighborhood similarity," in Proceedings of the 2010 IEEE International Symposium on A World of Wireless, Mobile and Multimedia Networks (WoWMoM), ser. WOWMOM '10,2010, pp. 1-9

[31] I.X.Y, Leung, P. Hui, P. Lio, and J. Crowcroft, "Towards real-time community detection in large networks," Phys. Rev. E, vol. 79, no. 6 Pt 2, p. 10, 2008.

[32] M. Chen, "Discovering communities by Information Diffusion", 2011 Eighth International Conference on Fuzzy Systems and Knowledge Discovery (FSKD).

[33] U.N. Raghavan, R. Albert, and S. Kumara, "Near linear time algorithm to detect community structures in large-scale networks," Phys. Rev. E, vol. 76, no. 3, p. 036106, 2007. 